\title{
openheart Management of perioperative myocardial ischaemia after isolated coronary artery bypass graft surgery
}

\author{
Davorin Sef, ${ }^{\oplus 1}$ Janko Szavits-Nossan, ${ }^{2,3}$ Mladen Predrijevac, ${ }^{3,4}$ Rajna Golubic, ${ }^{5}$ \\ Tomislav Sipic, ${ }^{2,3}$ Kresimir Stambuk, ${ }^{2,3}$ zvonimir korda, ${ }^{4}$ Pascal Meier, ${ }^{6,7}$ \\ Marko Ivan turina ${ }^{8}$
}

To cite: Sef D,

Szavits-Nossan J, Predrijevac M, et al. Management of perioperative myocardial ischaemia after isolated coronary artery bypass graft surgery. Open Heart 2019;6:e001027. doi:10.1136/ openhrt-2019-001027

Received 5 February 2019 Revised 4 April 2019 Accepted 12 April 2019

Check for updates

(c) Author(s) (or their employer(s)) 2019. Re-use permitted under CC BY-NC. No commercial re-use. See rights and permissions. Published by BMJ.

${ }^{1}$ Harefield Hospital, Harefield, UK 2Department of Cardiology, Magdalena - Clinic for Cardiovascular Diseases, Krapinske Toplice, Croatia ${ }^{3}$ J.J. Strossmayer University, Osijek, Croatia

${ }^{4}$ Department of Cardiovascular Surgery, Magdalena - Clinic for Cardiovascular Diseases, Krapinske Toplice, Croatia ${ }^{5}$ Papworth Hospital NHS Foundation Trust, Cambridge, , UK

${ }^{6}$ Royal Brompton and Harefield NHS Foundation Trust, London, UK

${ }^{7}$ Kantonsspital Graubunden, Chur, , Switzerland

${ }^{8}$ Universitat Zurich, Zurich, , Switzerland

Correspondence to Dr Davorin Sef; davorin.sef@ gmail.com

\section{ABSTRACT}

Objectives Updated knowledge about perioperative myocardial ischaemia (MI) after coronary artery bypass grafting $(\mathrm{CABG})$ and treatment of acute graft failure is needed. We analysed main factors associated with perioperative $\mathrm{Ml}$ and effects of immediate coronary angiography-based treatment strategy on patient outcome. Methods Among 1119 consecutive patients with coronary artery disease who underwent isolated CABG between January 2011 and December 2015, 43 (3.8\%) patients underwent urgent coronary angiography due to suspected perioperative Ml. All the data were prospectively collected and retrospectively analysed. The primary endpoint was 30-day mortality; postoperative left ventricular ejection fraction) and major adverse cardiac events were secondary endpoints.

\section{Results}

Overall, 30-day mortality in patients with CABG was $1.4 \%$ while in patients who developed perioperative $\mathrm{Ml}$ was $9 \%$ (4 patients). Angiographic findings included incorrect graft anastomosis, graft spasm, dissection, acute coronary artery thrombotic occlusion and ischaemia due to incomplete revascularisation. Emergency reoperation (Redo) was performed in 14 (32\%), acute percutaneous coronary intervention (PCl) in $15(36 \%)$ and conservative treatment (Non-op) in 14 patients. Demographic and preoperative clinical characteristics between the groups were comparable. Postoperative LVEF was significantly reduced in the Redo group ( $45 \%$ post-op vs $53 \%$ pre-op) and did not change in groups $\mathrm{PCl}(56 \%$ post-op vs $57 \%$ pre-op) and Non-op (58\% post-op vs $57 \%$ pre-op). Conclusions Urgent angiography allows identification of the various underlying causes of perioperative $\mathrm{Ml}$ and urgent treatment when this is needed. Urgent PCl may be associated with improved clinical outcome in patients with early graft failure.

\section{INTRODUCTION}

Perioperative myocardial infarction (MI) is one of the most serious complications following coronary artery bypass graft (CABG) surgery with reported incidence between $2 \%$ and $10 \%,{ }^{12}$ although the frequency varies depending on the method used for detection. Perioperative MI can be

\section{Key questions}

What is already known about this subject?

- Perioperative myocardial ischaemia (MI) is associated with an increased 30-day mortality in patients undergoing isolated coronary artery bypass grafting (CABG). However, additional information on the role of immediate angiography in the case of suspected perioperative $\mathrm{Ml}$ and the best treatment strategy would be helpful. There is still a lack of studies focused on types of bypass graft dysfunction.

What does this study add?

- In this study, we have analysed the main factors associated with perioperative $\mathrm{Ml}$ after isolated $\mathrm{CABG}$ surgery and effects of coronary angiography-based treatment strategy on the patient outcome. We report that the best treatment strategy should be discussed within the heart team to determine the optimal solution for each patient. However, our results demonstrated better clinical outcomes in patients who were treated with urgent percutaneous coronary intervention (PCl).

How might this impact on clinical practice?

- Urgent angiography allows identification of the various underlying causes of perioperative $\mathrm{MI}$ and urgent treatment when this is needed. However, urgent PCI may be associated with improved clinical outcome in patients with early graft failure.

related to graft-related causes (early graft failure, coronary artery thrombosis and postoperative spasm) along with non-graft related such as incomplete myocardial revascularisation, inadequate myocardial protection or preoperative ischaemic injury. ${ }^{2}{ }^{3}$ Early graft failure after CABG is reported in up to $12 \%$ of grafts ${ }^{4}$ (left internal mammary artery (LIMA) $1 \%-2.5 \%$; saphenous vein graft (SVG) $3 \%-12 \%$, radial artery $3 \%-4 \%$ ) when evaluated by intraoperative angiography; however, only a minority are symptomatic. ${ }^{5}$ Therefore, diagnosis of early graft failure is still a clinical challenge, particularly because elevation of cardiac biomarkers is ubiquitous after 
CABG and both ECG changes and echocardiographic wall motion abnormalities are not highly specific. ${ }^{3-9}$ To date, there is a lack of studies comparing the efficacy of percutaneous coronary intervention (PCI) versus redoCABG in patients with early graft failure. ${ }^{2} 8$ Current European Society of Cardiology/European Association for Cardio-Thoracic Surgery (ESC/EACTS) guidelines indicate immediate coronary angiography in the case of suspected perioperative MI to accurately detect the cause, enable an appropriate re-intervention strategy and to limit the extent of myocardial damage. ${ }^{5}$ However, this is based on level C evidence due to the limited data available. ${ }^{5}$ Early graft failure can be identified as the cause of perioperative MI in up to $82 \%$ of cases. ${ }^{10}$ Furthermore, the time interval between the onset of perioperative MI and re-intervention could have a considerable effect on both preservation of ventricular function and patient outcome. ${ }^{3}$ The purpose of this study was to analyse the main factors associated with perioperative MI after isolated CABG surgery and effects of coronary angiography-based treatment strategy on the patient outcome.

\section{MATERIALS AND METHODS Study design}

This study is a retrospective analysis of prospectively collected data at the Department of Cardiovascular Surgery in the Magdalena-Clinic for Cardiovascular Diseases in Croatia. Institutional ethic committee approval was obtained and all patients gave their written informed consent. Among 1119 patients with coronary artery disease who consecutively underwent isolated CABG between January 2011 and December 2015 for acute or stable coronary artery disease, $43(3.8 \%)$ patients underwent urgent coronary angiography due to suspected perioperative MI. Combined surgical procedures were excluded.

\section{Operative technique}

CABG surgery was performed through median sternotomy either on-pump (beating heart) or off-pump, according to the surgeon's preference, by a group of six surgeons. LIMA and SVG were the preferred grafts. Standard cardiopulmonary bypass (CPB) technique with beating heart and ascending aortic and two-stage venous cannulation under normothermic conditions was used in the cases of on-pump CABG. Intracoronary shunts were routinely used during on-pump CABG. Heparin was given to achieve an activated clotting time $>480 \mathrm{~s}$, except for off-pump CABG when it was maintained $>300 \mathrm{~s}$. Complete revascularisation, defined as bypass grafting to all coronary arteries $\geq 1.5 \mathrm{~mm}$ with a diameter reduction $\geq 50 \%$ in at least one angiographic view, ${ }^{11}$ was attempted in all cases. After completion of all anastomoses, anticoagulation was reversed with protamine sulfate.

\section{Perioperative care}

Routine medical history, physical examination and preoperative work-up with coronary angiography were done in all patients. Patients have received standard premedication including acetylsalicylic acid (ASA) at a daily dose of 75-325 mg until the day of the procedure, with anticoagulant drugs (warfarin) and adenosine diphosphate receptor (ADP) inhibitors (clopidogrel or ticagrelor) being discontinued 3-5 days before the procedure and replaced with subcutaneous low-molecular-weight heparin.

Standard monitoring (5-lead ECG, invasive arterial and venous pressures, pulse oximetry and rectal temperature) was used in all cases. Transoesophageal echocardiography (TEE) was selectively performed in the operating room by cardiac anaesthetist to evaluate myocardial and valvular function. TEE evaluation of regional wall motion abnormalities was assessed after the anaesthetic induction and after weaning from CPB. A medication of ASA (oral dose of $100 \mathrm{mg}$ ) was administered within the first 6-24 hours after the surgery in the absence of postoperative bleeding, followed by an oral maintenance dose of $100 \mathrm{mg}$ daily long term. In addition, clopidogrel was prescribed for 12 months (oral loading dose of $300 \mathrm{mg}$ and maintenance dose of $75 \mathrm{mg}$ ) at the discretion of operating surgeon. Subcutaneous low-molecular-weight heparin was started on the first postoperative day. Creatine kinase-muscle/brain (CK-MB) serum level measurements and 12-lead ECG were evaluated after CABG in the intensive care unit (ICU). These laboratory parameters were determined every 8 hours in the ICU and afterwards daily on the ward. Normal values were defined within the following ranges: CK 10-80 U/L, CK-MB $<5$ U/L. Perioperative MI was suspected after the detection of one of the following parameters: increase in the ratio of CK/CK-MB above 10\%, ischaemic ECG changes (ST-segment elevation or depression, development of new postoperative $Q$ wave, T-wave inversion and/or left bundle-branch block), recurrent episode of ventricular tachycardia or ventricular fibrillation, unexpected or uncontrolled haemodynamic deterioration or sudden cardiac arrest. Immediate angiography was indicated in the case of suspected perioperative MI and after a consultation within the heart team comprised of operating surgeon, anaesthetist or ICU physician and interventional cardiologist.

\section{Immediate angiographic control and treatment}

Angiographic findings including incorrect graft anastomosis, graft spasm, displacement, dissection, acute coronary artery thrombotic occlusion and ischaemia due to incomplete revascularisation were analysed. Angiographic examination included two-plane left ventriculography and multiple projections of both native coronary arteries and bypass graft. After consultation within the heart team and according to the clinical status and angiographic findings, patients underwent either emergency reoperation (Redo), acute PCI or conservative treatment (Non-op). Repeat PCI was performed at the time of diagnostic coronary angiography and in most of the cases included an intervention on the native coronary artery 
lesions in the area of the failing graft. After patients treated with PCI routinely received a dual antiplatelet therapy of ASA and clopidogrel (600 mg clopidogrel loading dose and $75 \mathrm{mg}$ clopidogrel maintenance dose with $100 \mathrm{mg}$ ASA daily long term) according to the ESC/The American Heart Association (AHA) guidelines. Emergency reoperation was performed in the case of anastomotic stenosis or occlusion when PCI was technically not feasible. Redo-CABG was performed with the same standard technique as in primary CABG. Conservative treatment included inotropic and mechanical support (intra-aortic balloon pump (IABP)) for haemodynamically compromised patients. Patients supported by IABP were heparinised intravenously maintaining activated partial thromboplastin time 1.5-2 times normal value. The primary endpoint was 30-day mortality. Postoperative left ventricular ejection fraction (LVEF\%), as assessed by transthoracic echocardiography, and major adverse cardiac event were secondary endpoints. Control postoperative echocardiography was performed before the discharge from hospital.

\section{Statistical analysis}

Categorical variables were tabulated as numbers with percentages. Continuous variables were shown as median values, with corresponding IQR. Differences in the categorical variables between the three treatment groups were tested using $\chi^{2}$ test or Fisher's exact test if the cell count was below 5 . Wilcoxon rank-sum test was used to test the significance of the difference in LVEF before and after the operation. Kruskall-Wallis test was conducted to test the differences in continuous variables between the groups. Non-parametric tests were used due to relatively small sample size and departure from normal distribution.

\section{RESULTS}

Perioperative MI was suspected in 43 patients (12 women and 31 men). Immediate PCI was performed in 15 (36\%), redo-CABG in $14(32 \%)$ and conservative treatment in 14 patients (32\%). Preoperative characteristics of the patients are demonstrated in table 1 and no statistically

\section{Table 1 Preoperative characteristics of the patients}

\begin{tabular}{|c|c|c|c|c|}
\hline & Redo (N=14) & $\mathrm{PCl}(\mathrm{N}=15)$ & Non-op ( $\mathrm{N}=14)$ & $P$ value \\
\hline $\begin{array}{l}\text { Age (y), median (IQR) } \\
\text { Gender }\end{array}$ & $64.0(52.5,69.8)$ & $64.0(59.0,70.0)$ & $63(59.0,67.0)$ & 0.621 \\
\hline Men, N (\%) & $9(64)$ & $11(73)$ & $11(79)$ & \\
\hline Women, N (\%) & $5(36)$ & $4(27)$ & $3(21)$ & 0.768 \\
\hline LVEF (\%), median (IQR) & $60(40,65)$ & $60(55,65)$ & $60(60,65)$ & 0.834 \\
\hline EuroSCORE II, median (IQR) & $1.7(1.3,5.0)$ & $1.0(0.5,6.4)$ & $1.0(0.7,2.1)$ & 0.193 \\
\hline \multicolumn{5}{|l|}{ HA } \\
\hline Yes, N (\%) & $11(79)$ & $12(80)$ & $14(100)$ & 0.216 \\
\hline \multicolumn{5}{|l|}{ DM } \\
\hline Yes, N (\%) & $4(29)$ & $4(27)$ & $7(50)$ & 0.446 \\
\hline \multicolumn{5}{|l|}{ HLP } \\
\hline Yes, N (\%) & $6(43)$ & $5(33)$ & $8(57)$ & 0.472 \\
\hline \multicolumn{5}{|l|}{ COPD } \\
\hline Yes, $N(\%)$ & $2(14)$ & $2(13)$ & $4(29)$ & 0.618 \\
\hline \multicolumn{5}{|l|}{ Chronic kidney disease } \\
\hline Yes, N (\%) & $4(29)$ & $3(20)$ & $2(14)$ & 0.733 \\
\hline \multicolumn{5}{|l|}{ Previous PCl } \\
\hline Yes, N (\%) & $11(79)$ & $13(87)$ & 12(86) & 0.883 \\
\hline \multicolumn{5}{|l|}{ Previous Ml } \\
\hline Yes, N (\%) & $9(64)$ & $5(33)$ & $9(64)$ & 1.000 \\
\hline No of diseased vessels $\geq 2$ or left main stenosis, $\mathrm{N}(\%)$ & $13(93)$ & $14(93)$ & $13(93)$ & 1.000 \\
\hline \multicolumn{5}{|l|}{ Operative urgency } \\
\hline Elective, $\mathrm{N}(\%)$ & $8(57)$ & $9(60)$ & $9(64.3)$ & \\
\hline Urgent, $\mathrm{N}(\%)$ & $5(36)$ & $4(27)$ & $4(28.6)$ & \\
\hline Emergent, N (\%) & $1(7)$ & $2(13)$ & $1(7.1)$ & 1.000 \\
\hline
\end{tabular}

COPD, chronic obstructive pulmonary disease; DM, diabetes mellitus; HA, arterial hypertension; HLP, hypelipoproteinaemia; LVEF, left ventricular ejection fraction; $\mathrm{PCl}$, percutaneous coronary intervention; $\mathrm{Y}$, year. 
Table 2 Perioperative characteristics of the patients

\begin{tabular}{|c|c|c|c|c|}
\hline & Redo (N=14) & $\mathrm{PCl}(\mathrm{N}=15)$ & Non-op $(\mathrm{N}=14)$ & $P$ value \\
\hline CK-MB postop (U/L), mean (SD) & $141.9(130.1)$ & $52.7(35.2)$ & $52.6(40.6)$ & 0.009 \\
\hline Intubation time (h), median (IQR) & $30(18,48)$ & $28(13,65)$ & $15.5(10.0,20)$ & 0.141 \\
\hline LVEF (\%), median (IQR), postoperative & $45(35,55)$ & $60 \%(50,65)$ & $60(55,65)$ & 0.006 \\
\hline \multicolumn{5}{|l|}{ CABG } \\
\hline On-pump, N(\%) & $8(57)$ & $2(13)$ & $5(36)$ & \\
\hline Off-pump, N(\%) & $6(43)$ & $13(87)$ & $9(64)$ & 0.047 \\
\hline \multicolumn{5}{|l|}{ ECG changes } \\
\hline Positive, N(\%) & $9(64)$ & $12(80)$ & $6(57)$ & 0.432 \\
\hline \multicolumn{5}{|l|}{ Chronic haemodialysis } \\
\hline Yes, $N(\%)$ & $1(7)$ & 0 & 0 & 0.651 \\
\hline \multicolumn{5}{|l|}{ IABP } \\
\hline Yes, N (\%) & $12(86)$ & $6(40)$ & $2(14)$ & 0.001 \\
\hline \multicolumn{5}{|l|}{ VF (CPR) } \\
\hline Yes, $N(\%)$ & $3(21)$ & $2(13)$ & 0 & 0.256 \\
\hline \multicolumn{5}{|l|}{$\mathrm{MOF}$} \\
\hline Yes, $N(\%)$ & $4(29)$ & 0 & 0 & 0.016 \\
\hline \multicolumn{5}{|l|}{ 30-day mortality } \\
\hline Yes, N (\%) & $4(29)$ & 0 & 0 & 0.016 \\
\hline
\end{tabular}

CABG, coronary artery bypass grafting; CK-MB, creatine kinase-muscle/brain; CPR, cardiopulmonary resuscitation; IABP, intra-aortic baloon pump; LVEF, left ventricular ejection fraction; MOF, multiorgan failure; VF, ventricular fibrilation.

significant difference was found between the three treatment groups. Over $90 \%$ of the patients in each group had two or three vessel disease. Approximately one-third of the operations was carried out as emergencies and did not significantly differentiate between the groups $(\mathrm{p}=1.000)$.

The Redo group exhibited the highest peak CK-MB level during first 24 hours postoperatively with mean of $141.9 \pm 130.1 \mathrm{U} / \mathrm{L}$ compared with $52.7 \pm 35.2 \mathrm{U} / \mathrm{L}$ in the PCI group and 52.6 $\pm 40.6 \mathrm{U} / \mathrm{L}(\mathrm{p}=0.009)$ in the Non-op group (table 2, figure 1). Global postoperative LVEF was substantially lower in the Redo group compared with the PCI and the Non-op group, with a mean of $45 \%, 56 \%$ and $58 \%$, respectively ( $\mathrm{p}=0.006$, Kruskal-Wallis test). The magnitude of the difference in the LVEF after and before the operation was substantially higher in the Redo group when compared with other groups with a mean of 53\% preoperatively versus $45 \%$ postoperatively (figure 1 ). However, this was not statistically significant $(\mathrm{p}=0.110$, Wilcoxon rank-sum test).

Overall, 30-day mortality in patients with CABG was 1.4\% while in patients who developed perioperative MI was 9\% (4 patients) (table 2). All these four patients occurred in the Redo group (29\%). In contrast, there were no deaths in the other two groups $(\mathrm{p}=0.016)$. The same pattern was observed for multiorgan failure (MOF) with all four cases (29\%) occurring in the Redo group. Furthermore, the Redo group also showed significantly greater need for perioperative IABP $(\mathrm{N}=12,86 \%)$ compared with the PCI $(\mathrm{N}=6,40.0 \%)$ and the Non-op group $(\mathrm{N}=2,14 \% ; \mathrm{p}=0.001)$. Significantly greater proportion of patients in the Redo group had the primary $\mathrm{CABG}$ performed on-pump $(\mathrm{N}=8$, $57 \%)$ compared with the PCI $(\mathrm{N}=2,13 \%)$ and the Non-op group $(\mathrm{N}=5,36 \% ; \mathrm{p}=0.047$.

Overall, graft dysfunction was observed in 31 patients (72\%) (table 3). In the Redo group, 11 patients (79\%) had a single graft failure which was comparable to the PCI group ( $\mathrm{N}=11,73 \%)$. In contrast, only two patients $(14 \%)$ in the Non-op group had a single graft failure. There was a statistically significant difference in the type of graft failure by group $(\mathrm{p}<0.001)$. The most commonly failed graft in the Redo group was LIMA ( $\mathrm{N}=7,43.8 \%)$ while in the PCI group it was SVG $(\mathrm{N}=11,68.8 \%)$. In the Non-op group, there were only two patients $(14 \%)$ with a graft failure and it was SVG in both cases. However, in

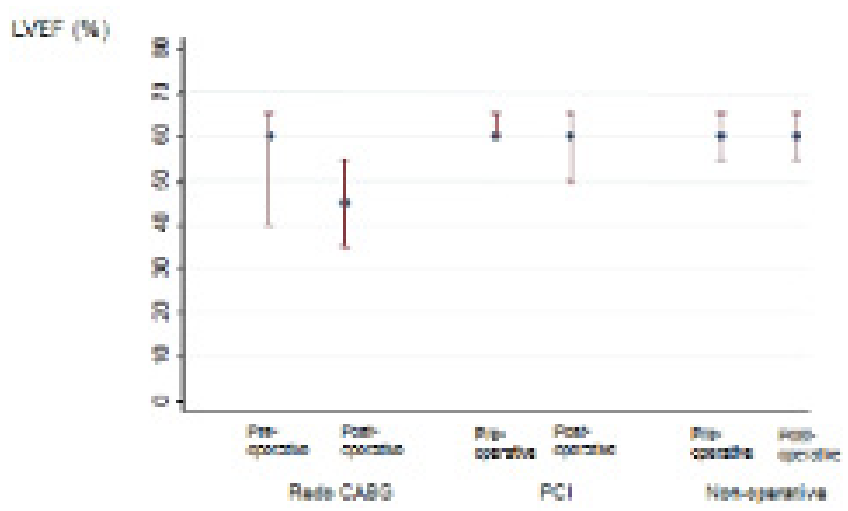

Figure 1 Median (IQR) preoperative versus postoperative LVEF (\%) by group. LVEF, left ventricular ejection fraction. 
Coronary artery disease

Table 3 Postoperative characteristics of the patients

\begin{tabular}{|c|c|c|c|c|}
\hline & Redo ( $N=14)$ & $\mathrm{PCl}(\mathrm{N}=15)$ & Non-op ( $\mathrm{N}=14)$ & $P$ value \\
\hline \multicolumn{5}{|c|}{ Number of primary bypass grafts, $\mathrm{N}(\%)$} \\
\hline 1 & $1(7.1)$ & $1(6.6)$ & $1(7.1)$ & \\
\hline 2 & $7(50.0)$ & $7(46.7)$ & $9(64.4)$ & \\
\hline 3 & $6(42.9)$ & $7(46.7)$ & $4(28.4)$ & 0.910 \\
\hline \multicolumn{5}{|l|}{ Number of failing grafts, $\mathrm{N}(\%)$} \\
\hline 1 & $11(78.6)$ & $11(73.4)$ & $2(14.2)$ & \\
\hline 2 & $2(14.2)$ & $4(26.6)$ & 0 & \\
\hline 3 & $1(7.1)$ & 0 & 0 & $\mathrm{p}<0.001$ \\
\hline \multicolumn{5}{|l|}{ Failing graft, $\mathrm{N}(\%)^{*}$} \\
\hline LIMA & $7(43.8)$ & $4(25)$ & 0 & \\
\hline SVG & $6(37.5)$ & $11(68.8)$ & $2(14.2)$ & \\
\hline SVG and LIMA & $2(14.2)(12.5)$ & $1(6.2)$ & 0 & \\
\hline RIMA & $1(6.2)$ & 0 & 0 & $p<0.001$ \\
\hline \multicolumn{5}{|l|}{ Timing of postoperative angiography } \\
\hline & 7 hour $21 \mathrm{~min}$ & 29 hours $18 \mathrm{~min}$ & 15 hours $20 \mathrm{~min}$ & $p<0.001$ \\
\hline \multicolumn{5}{|l|}{ Type of graft failure, $\mathrm{N}(\%)^{*}$} \\
\hline Graft occlusion & $5(27.8)$ & 0 & 0 & \\
\hline Graft thrombosis & $6(33.3)$ & $8(42.1)$ & $1(7.1)$ & \\
\hline Anastomosis stenosis $(>50 \%)$ & $6(33.3)$ & $9(47.4)$ & $1(7.1)$ & \\
\hline Dissection & $1(5.6)$ & 0 & 0 & \\
\hline Spasm & 0 & 0 & 0 & \\
\hline Incomplete revascularisation & 0 & $2(10.5)$ & 0 & $\mathrm{p}<0.001$ \\
\hline \multicolumn{5}{|l|}{ Graft failure, $\mathrm{N}(\%)$} \\
\hline Yes & $14(100)$ & $15(100)$ & $2(14.2)$ & $\mathrm{p}<0.001$ \\
\hline \multicolumn{5}{|c|}{ Affected target coronary arteries, $\mathrm{N}(\%)^{*}$} \\
\hline LAD & $12(66.7)$ & $11(64.7)$ & 0 & \\
\hline $\mathrm{Cx} / 0 \mathrm{M}$ & $4(22.2)$ & $2(11.8)$ & $1(7.1)$ & \\
\hline RCA/PDA & $2(11.1)$ & $4(23.5)$ & $1(7.1)$ & $\mathrm{p}<0.001$ \\
\hline \multicolumn{5}{|l|}{ Re-intervention, $\mathrm{N}(\%)^{*}$} \\
\hline One vessel & $11(78.6)$ & $11(73.4)$ & 0 & \\
\hline Ttwo vessels & $2(14.2)$ & $4(26.6)$ & 0 & \\
\hline Tthree vessels & $1(7.1)$ & 0 & 0 & \\
\hline Hybrid & $1(7.1)$ & 0 & 0 & $p<0.001$ \\
\hline \multicolumn{5}{|l|}{ Type of intervention, $\mathrm{N}(\%)$} \\
\hline Additional bypass grafting & $13(92.9)$ & 0 & $\mathrm{~N} / \mathrm{A}$ & \\
\hline Re-anastomosis & $1(7.1)$ & 0 & $\mathrm{~N} / \mathrm{A}$ & \\
\hline Native vessel PCl & 0 & $14(93.8)$ & $\mathrm{N} / \mathrm{A}$ & \\
\hline Bypass graft PCl & 0 & $1(6.2)$ & $\mathrm{N} / \mathrm{A}$ & $p<0.001$ \\
\hline
\end{tabular}

${ }^{*}$ The percentages in the parentheses always reflect the percentage in the corresponding column.

Cx, circumflex coronary artery; LAD, left anterior descending artery; LIMA, indicates left internal mammary artery; OM, marginal branch of Cx; $\mathrm{PCI}$, percutaneous coronary intervention; PDA, posterior descending artery; RCA, right coronary artery; RIMA, right internal mammary artery; SVG, saphenous vein graft.

these two patients due to technical reasons and clinical evidence of improvement after initial conservative treatment, decision was made to continue with the same treatment. In the Redo group, graft thrombosis and stenosis of anastomosis showed the same frequency $(\mathrm{N}=6,33.3 \%)$ while in the PCI group the stenosis of the anastomosis was the predominant type $(\mathrm{N}=9,47.4 \% ; \mathrm{p}=0.001)$. All patients with normal graft findings were in the Non-op 
group $(\mathrm{N}=12,86 \%)$. The most frequently affected coronary artery was left anterior descending artery in both the Redo (66.7\%) and the PCI group (64.7\%). The difference in the number of patients with affected coronaries between the groups was not statistically significant $(p=0.275)$. In the PCI group, 93.3\% of the patients were successfully treated and only one patient needed a second re-intervention.

\section{DISCUSSION}

Early graft failure after CABG surgery complicated by perioperative MI can be associated with a dismal prognosis. $^{2}$ Several studies supported the beneficial effects of early coronary angiography with reintervention in patients with graft-related causes of perioperative MI. ${ }^{3812}$ However, only few studies were focused on the type of graft dysfunction. ${ }^{8} 10$ Patients with significant haemodynamic compromise (arrhythmias, cardiogenic shock) were obviously immediately taken to the operating room for potential correction. If postoperative derangement was reasonably well tolerated, angiography and/or PCI were performed. One of the major findings of this study is that when perioperative MI was suspected, graft dysfunction was confirmed by immediate angiographic control in $72 \%$ patients. Control angiography accurately differentiated a significant minority of patients in whom the grafts were patent and could be optimised conservatively. We observed better outcome in the group of patients treated with emergency PCI after early graft failure, if technically feasible, than in the group of patients who underwent reoperation. We observed no 30-day mortality and no decline in postoperative left ventricle function in the PCI group. The Redo group exhibited the highest mortality with four patients not surviving surgery, but all these four patients were severely haemodynamically compromised and developed MOF. As a result of the critical condition of these patients, we observed a significantly shorter period between primary surgery and postoperative angiography in the Redo group. We can explain these findings as these patients had the most severe myocardial damage having the highest increase in CK-MB perioperatively and thus were put at a higher risk for redo-CABG.

The detection of perioperative MI following isolated CABG surgery still remains a challenge. ${ }^{10}{ }^{12-14}$ In most of the cases, perioperative MI is caused by a variety of graft-related causes such as technical errors and graft spasm. $^{23}$ The majority of these complications can be amendable surgically or with PCI, particularly if treated on time, in order to reduce the extent of myocardial damage and improve clinical outcome. To date, there is still sparse literature focusing on management of early graft failure following CABG surgery. Recent studies are limited by the lack of a control cohort of patients treated conservatively and non-randomisation of different treatment groups. La Flamme et al reported that early re-intervention may be superior to conservative treatment but did not compare different types of re-intervention. ${ }^{10}$
However, they observed 30-day mortality of $15.8 \%$ in the re-intervention group and $15.4 \%$ in the conservatively treated group of patients and found no significant difference between the two groups, although they excluded emergent cases. Davierwala et al showed overall 30-day mortality of $7.3 \%$ and found that morbidity and 30-day mortality $(10.8 \%)$ were significantly higher in patients who underwent revision CABG for perioperative MI when compared with PCI (5.9\%). However, this was a retrospective study including a significant proportion (25.5\%) of patients with incomplete revascularisation. In both studies, different types of graft dysfunction were not analysed. Thielmann et al observed 30-day mortality of $12 \%$ in patients with PCI re-intervention, 20\% after emergency reoperation and $14.8 \%$ among patients treated conservatively. However, this study enrolled only patients with early graft failure diagnosed within first 24 hours after surgery and excluded emergency surgery.

In our study, 12 patients (28\%) with suspected perioperative MI had normal grafts on control angiography. This result highlights the importance of performing immediate control angiography, if possible, whenever perioperative MI is suspected prior to considering redoCABG surgery. Furthermore, we have not observed any significant increase in acute renal failure related to repeat angiography. However, the most common method of assessing graft patency intraoperatively is a transit-time flow measurement and it can provide a possible solution for intraoperative graft failure, although this is of limited value and less accurate than angiography. ${ }^{15-17}$ Primary success rate in the PCI group was excellent with only one patient needing a second re-intervention. Interestingly, we found no significant difference in the number of failing grafts between PCI and Redo groups neither in affected target coronary arteries. Our findings suggest that the choice of treatment was principally based on the type of graft failure and clinical condition of the patient. In addition, in the Redo group LIMA grafts demonstrated the highest frequency of failing, while in the PCI group this was observed for the SVG. As reported in the literature, it may be technically easier to perform PCI when the failed graft is SVG. ${ }^{18}$ Interestingly, we found that PCI group had the highest proportion of CABG surgery performed off-pump but we could not provide an explanation for this.

Overall 30-day mortality in our study was comparable or even less than in recently published studies. ${ }^{2810} 1920$ In a recently published meta-analysis that included 1104 patients with perioperative MI after CABG, overall 30-day mortality was $8.9 \% .{ }^{19}$ Furthermore, Preußer et al analysed mid-term results and reported survival rates at 7 years of $62.5 \%$ in the perioperative MI group and $81.1 \%$ in non-perioperative MI group. ${ }^{20}$

One of the limitations of this study is that these three groups (PCI, Redo and Non-op) are truly not comparable. Even though group baseline characteristics are comparable, a selection bias is possible as the choice of treatment was not randomised but we decided for the best 
treatment option individualised to each patient based on clinical situation and angiographic findings. Therefore, we could not draw a conclusion that PCI treatment is a better choice than redo-CABG, although we observed better outcome in the PCI group. In addition, we have not utilised transit-time flow measurement in our current practice which would probably decrease the number of perioperative MI diagnosed after the surgery. Furthermore, we do not think that including emergency patients can be misleading, as diagnostic criteria for perioperative MI are well established (ignoring isolated postsurgical elevations of cardiac biomarkers). As well, trends in ECG changes and CK-MB level were considered while distinguishing preoperative from perioperative MI.

\section{CONCLUSIONS}

Perioperative MI is associated with an increased 30-day mortality in patients undergoing isolated CABG. Urgent angiography allows identification of the various underlying causes of perioperative MI and urgent treatment when this is needed. However, urgent PCI may be associated with improved clinical outcome in patients with early graft failure.

\section{Contributors The authors of the paper take full responsibility.}

Funding The authors have not declared a specific grant for this research from any funding agency in the public, commercial or not-for-profit sectors.

Competing interests None declared.

Patient consent for publication Not required.

Ethics approval Ethics Committee of Magdalena - Clinic for Cardiovascular Diseases.

Provenance and peer review Not commissioned; externally peer reviewed. Data sharing statement Data are available in a public, open access repository.

Open access This is an open access article distributed in accordance with the Creative Commons Attribution Non Commercial (CC BY-NC 4.0) license, which permits others to distribute, remix, adapt, build upon this work non-commercially, and license their derivative works on different terms, provided the original work is properly cited, appropriate credit is given, any changes made indicated, and the use is non-commercial. See: $\mathrm{http}: / /$ creativecommons.org/licenses/by-nc/4.0/.

\section{REFERENCES}

1. Mehta RH, Ferguson TB, Lopes RD, et al. Saphenous vein grafts with multiple versus single distal targets in patients undergoing coronary artery bypass surgery: one-year graft failure and five-year outcomes from the project of ex-vivo vein graft engineering via transfection (prevent) IV trial. Circulation 2011;124:280-8.

2. Davierwala PM, Verevkin A, Leontyev S, et al. Impact of expeditious management of perioperative myocardial ischemia in patients undergoing isolated coronary artery bypass surgery. Circulation 2013;128(11 Suppl 1):S226-S234.

3. Szavits-Nossan J, Stipić H, Sesto I, et al. Angiographic control and percutaneous treatment of myocardial ischemia immediately after CABG. Coll Antropol 2012;36:1391-4.
4. Zhao DX, Leacche M, Balaguer JM, et al. Routine intraoperative completion angiography after coronary artery bypass grafting and 1-stop hybrid revascularization results from a fully integrated hybrid catheterization laboratory/operating room. J Am Coll Cardiol 2009;53:232-41.

5. Windecker S, Kolh P, Alfonso F, Alfonso F, et al. 2014 ESC/EACTS Guidelines on myocardial revascularization: The Task Force on Myocardial Revascularization of the European Society of Cardiology (ESC) and the European Association for Cardio-Thoracic Surgery (EACTS)Developed with the special contribution of the European Association of Percutaneous Cardiovascular Interventions (EAPCI). Eur Heart J 2014;35:2541-619.

6. Fukui T. Intraoperative graft assessment during coronary artery bypass surgery. Gen Thorac Cardiovasc Surg 2015;63:123-30.

7. Narayan P, Bandyopadhyay M, Khan MW, et al. Angiography for management of perioperative myocardial ischemia: does it have any role? Innovations 2014;9:375-8. discussion 8.

8. Thielmann M, Massoudy P, Jaeger BR, et al. Emergency rerevascularization with percutaneous coronary intervention, reoperation, or conservative treatment in patients with acute perioperative graft failure following coronary artery bypass surgery. Eur J Cardiothorac Surg 2006;30:117-25.

9. Nielsen S, Björck L, Jeppsson A, et al. Trends in mortality risks among 94,328 patients surviving 30 days after a first isolated coronary artery bypass graft procedure from 1987 to 2006: a population-based study. Int J Cardiol 2017;244:316-21.

10. Laflamme M, DeMey N, Bouchard D, et al. Management of early postoperative coronary artery bypass graft failure. Interact Cardiovasc Thorac Surg 2012;14:452-6.

11. Farooq V, Serruys PW, Garcia-Garcia HM, et al. The negative impact of incomplete angiographic revascularization on clinical outcomes and its association with total occlusions: the SYNTAX (synergy between percutaneous coronary intervention with Taxus and cardiac surgery) trial. J Am Coll Cardiol 2013;61:282-94.

12. Alter $\mathrm{P}$, Vogt $\mathrm{S}$, Herzum $\mathrm{M}$, et al. Indications for angiography subsequent to coronary artery bypass grafting. Am Heart $J$ 2005;149:1082-90.

13. Bassiri $\mathrm{H}$, Nematollahi $\mathrm{A}$, Noohi $\mathrm{F}$, et al. Coronary graft patency after perioperative myocardial infarction: a study with multislice computed tomography. Interact Cardiovasc Thorac Surg 2011;12:596-9

14. Moussa ID, Klein LW, Shah B, et al. Consideration of a new definition of clinically relevant myocardial infarction after coronary revascularization: an expert consensus document from the Society for cardiovascular angiography and interventions (Scal). Catheter Cardiovasc Interv 2014;83:27-36.

15. Becit N, Erkut B, Ceviz M, et al. The impact of intraoperative transit time flow measurement on the results of on-pump coronary surgery. Eur J Cardiothorac Surg 2007;32:313-8.

16. Hirotani T, Kameda T, Shirota S, et al. An evaluation of the intraoperative transit time measurements of coronary bypass flow. Eur J Cardiothorac Surg 2001;19:848-52.

17. Chin ASL, Goldman LE, Eisenberg MJ. Functional testing after coronary artery bypass graft surgery: a meta-analysis. Can J Cardiol 2003;19:802-8

18. Sen H, Lam MK, Tandjung K, et al. Impact of previous coronary artery bypass surgery on clinical outcome after percutaneous interventions with second generation drug-eluting stents in TWENTE trial and Non-Enrolled TWENTE registry. Int $\mathrm{J}$ Cardiol 2014;176:885-90.

19. Biancari F, Anttila V, Dell'Aquila AM, et al. Control angiography for perioperative myocardial ischemia after coronary surgery: metaanalysis. J Cardiothorac Surg 2018;13.

20. Preußer MJ, Landwehrt J, Mastrobuoni S, et al. Survival results of postoperative coronary angiogram for treatment of perioperative myocardial ischaemia following coronary artery bypass grafting: a single-centre experience. Interact Cardiovasc Thorac Surg 2018;26:237-42. 